\title{
High-Specific Impulse Hall Thrusters, Part 2: Efficiency Analysis
}

\author{
Richard R. Hofer* \\ QSS Group, Inc., Cleveland, Ohio 44135 \\ and \\ Alec D. Gallimore ${ }^{\dagger}$ \\ University of Michigan, Ann Arbor, Michigan 48109
}

\begin{abstract}
Performance and plasma measurements of a high-specific impulse (2000-3000 s) Hall thruster were analyzed using a phenomenological performance model that accounted for a partially ionized plasma containing multiply charged ions. Anode efficiency over discharge voltages of 300-900 V ranged from 57 to 69\%, which corresponded to 89-97\% voltage utilization, 86-90\% mass utilization, 77-81\% current utilization, and 97-99\% charge utilization. Although the net decrease of efficiency due to multiply charged ions was at most $3 \%$, the effects of multiply charged ions on the discharge current could not be neglected because the increase of the discharge current with voltage was primarily due to the increasing fraction of multiply charged ions. This and the fact that the maximum deviation of the electron current from its average value was only $+5 /-14 \%$ illustrated how efficient operation at high-specific impulse was enabled through the regulation of the electron current with the applied magnetic field. The electron Hall parameter, defined by acceleration zone plasma properties, was nearly constant with voltage, decreasing from an average of 210 at $300 \mathrm{~V}$ to an average of 160 between 400 to $900 \mathrm{~V}$.
\end{abstract}

$\begin{array}{lll}A & = & \text { cross-sectional area of the annular discharge } \\ & & \text { chamber } \\ B_{r} & = & \text { radial component of the magnetic field } \\ B_{r, \text { max }} & = & \text { maximum radial magnetic field on discharge } \\ & & \text { chamber centerline } \\ B_{0} & = & \text { magnetic field normalization constant } \\ E_{z} & = & \text { axial component of the electric field } \\ e & = & \text { electron charge } \\ I_{b} & = & \text { ion beam current, } \Sigma I_{i} \\ I_{d} & = & \text { discharge current, } I_{b}+I_{e} \\ I_{e} & = & \text { axial electron current } \\ I_{i} & = & \text { current of the } i \text { th ion species } \\ i & = & \text { ion charge-stage index, } 1,2,3, \text { etc. } \\ j_{e z}, j_{e \theta} & = & \text { axial and azimuthal electron current densities } \\ \dot{m}_{a}, \dot{m}_{c} & =\text { anode and cathode mass flow rates } \\ \dot{m}_{b} & =\text { ion beam mass flow rate, } \Sigma \dot{m}_{i} \\ \dot{m}_{i} & =\text { mass flow rate of the } i \text { th ion species } \\ \dot{m}_{t} & =\text { total mass flow rate, } \dot{m}_{a}+\dot{m}_{c} \\ m_{x e} & =\text { mass of a xenon atom } \\ N & =\text { total number of ion species } \\ n_{b} & =\text { ion beam number density, } \Sigma n_{i} \\ n_{e} & =\text { electron number density } \\ n_{i} & =\text { number density of the } i \text { th ion species } \\ P_{d} & =\text { discharge power, } V_{d} I_{d} \\ P_{\text {jet }} & =\text { jet (or beam) power, } T^{2} / 2 \dot{m}_{t} \\ & \end{array}$

Presented as Paper 2004-3602 at the AIAA/ASME/SAE/ASEE 40th Joint Propulsion Conference and Exhibit, Fort Lauderdale, FL, 11-14 July 2004; received 4 February 2005; revision received 9 December 2005; accepted for publication 3 January 2006. Copyright (C) 2005 by the American Institute of Aeronautics and Astronautics, Inc. The U.S. Government has a royaltyfree license to exercise all rights under the copyright claimed herein for Governmental purposes. All other rights are reserved by the copyright owner. Copies of this paper may be made for personal or internal use, on condition that the copier pay the $\$ 10.00$ per-copy fee to the Copyright Clearance Center, Inc., 222 Rosewood Drive, Danvers, MA 01923; include the code 0748-4658/06 \$10.00 in correspondence with the CCC.

${ }^{*}$ Research Engineer, Electric Propulsion Branch, NASA Glenn Research Center; currently Member of the Technical Staff, Mail Stop 125-109, Advanced Propulsion Technology Group, Propulsion and Materials Engineering Section, Jet Propulsion Laboratory, 4800 Oak Grove Drive, Pasadena, CA 91109; richard.r.hofer@jpl.nasa.gov. Member AIAA.

${ }^{\dagger}$ Professor, Department of Aerospace Engineering, Plasmadynamics and Electric Propulsion Laboratory, 3037 François-Xavier Bagnoud Building, 1320 Beal Avenue; alec.gallimore@umich.edu. Associate Fellow AIAA.

$P_{\mathrm{mag}}$
$P_{t}$
$T$
$V_{a}$
$V_{d}, V_{l}$
$V_{\mathrm{mp}}$
$V_{p}$
$V_{\mathrm{rpa}}, V_{\mathrm{true}}$
$v_{\mathrm{ExB}}$
$Z_{i}$
$\varepsilon$
$\zeta_{i}$
$\eta_{a}, \eta_{b}$
$\eta_{c}$
$\eta_{\mathrm{mag}}$
$\eta_{m}, \eta_{q}, \eta_{v}$
$\eta_{t}$
$\xi$
$\Omega_{e}$
$\Omega_{i}$

$=$ electromagnet coil power

$=$ total input power, $P_{d}+P_{\mathrm{mag}}$

$=$ thrust

average acceleration voltage, $V_{d}-V_{l}$

discharge and ion loss voltages

most-probable ion voltage, $\approx V_{a}$

plasma potential

uncorrected and corrected ion voltages

ExB drift velocity

charge-state of the $i$ th ion species, 1,2,3, etc.

electron current fraction, $I_{e} / I_{d}$

fraction of the $i$ th ion species, $n_{i} / n_{b}$

anode and current utilization efficiencies

cathode efficiency, $\dot{m}_{a} / \dot{m}_{t}$

electromagnet coil efficiency, $P_{d} / P$

mass, charge, and voltage utilization efficiencies total efficiency

exchange ratio, $m_{x e} I_{d} / \dot{m}_{a} e$

$=$ electron Hall parameter

$=$ current fraction of the $i$ th ion species, $I_{i} / I_{b}$

\section{Introduction}

$\mathbf{W}$ ESTERN interest in Russian Hall thruster technology emerged in the 1990s partly because the 1600 -s specific impulse characteristic of flight hardware fell between arcjets (less than $1000 \mathrm{~s}$ ) and gridded ion thrusters (greater than $3000 \mathrm{~s}$ ) (Refs. 1 and 2). After Russian Hall thrusters met western flight qualification standards in the mid-1990s, interest in expanding the specific impulse range developed, mostly toward $3000 \mathrm{~s}$, so that the Hall thruster could more fully bridge the $1000-3000$ s specific impulse gap. These activities were motivated by studies indicating the mission benefits for Earth orbiting ${ }^{2-7}$ and interplanetary ${ }^{7-11}$ spacecraft if Hall thrusters could operate efficiently at high-specific impulse (2000$3000 \mathrm{~s}$ ). These benefits have included greater payloads, launch vehicle step downs, reduced trip times, or lower required power. Because the realization of these benefits depends on high-specific impulse Hall thrusters being available, a clear need exists to explore the development challenges of such technology enhancements.

Beginning in the late 1990 s, the feasibility of operating modern, xenon Hall thrusters at high-specific impulse was investigated. Although the initial studies of commercially developed Hall thrusters demonstrated greater than 4000-s specific impulse, maximum thruster efficiency occurred at less than 3000 s (Refs. 12-14). 
Follow-on efforts focused on the development and characterization of xenon Hall thrusters capable of both high-specific impulse and high-efficiency operation. ${ }^{15-22}$ The research resulted in the NASA$173 \mathrm{M}$ series of laboratory-model Hall thrusters.

The designs of the NASA-173M series thrusters were based on the hypothesis that the efficiency maximum observed with the commercially developed thrusters was actually a consequence of modern magnetic field designs that have been optimized for 1600-s specific impulse. The new thrusters were, therefore, an attempt to improve efficiency through the implementation of a magnetic circuit intended for high-specific impulse operation. The basis of the magnetic field design was a topography shaped as a plasma lens. ${ }^{22}$

The role of the magnetic field in achieving efficient operation can be shown by considering the relationship between the electron Hall parameter, the axial electron current, and the magnetic field. First, as a basic operating principle of Hall thrusters the electron Hall parameter satisfies

$$
\Omega_{e}=j_{e \theta} / j_{e z} \gg 1
$$

In this form, the Hall parameter characterizes the number of azimuthal orbits that an electron executes before being perturbed by particle collisions, collisions with the walls, or plasma fluctuations. The high value of the Hall parameter, being much greater than unity but not infinite, implies that electrons must undergo a sufficient number of ionizing collisions on their way to the anode to sustain the plasma discharge while still maintaining a bulk azimuthal current that is several times larger than the axial current. When this argument is taken one step further, it is reasonable to expect that efficient Hall thruster operation can only be achieved over a narrower range of Hall parameters than which a stable discharge can be achieved.

The relationship between the Hall parameter and thruster efficiency can be shown by considering the current utilization efficiency. The current utilization efficiency, which largely determines the anode efficiency, is given by

$$
\eta_{b}=I_{b} / I_{d}=1 /\left[1+\left(I_{e} / I_{b}\right)\right]
$$

For constant discharge current, Eq. (2) shows how minimizing the electron current increases the current utilization by maximizing the ion yield. The electrons also largely determine the ionization efficiency (through their average temperature) and the acceleration efficiency (by establishing the self-consistent electric field). Clearly, an understanding of electron dynamics is vital in the study of Hall thruster physics.

Unfortunately, the physics determining the axial electron current in a Hall thruster is a seemingly intractable problem that has plagued researchers for decades. ${ }^{23-30}$ This is mainly due to the presence of anomalous diffusion mechanisms that increase the electron crossfield transport beyond that predicted by classical transport theory. The two leading candidates thought to be responsible for anomalous transport are collisions with the walls and turbulent plasma fluctuations. Determining which of these mechanisms is dominant has been the subject of considerable debate since the 1960s. A review in Ref. 21 of past experimental, numerical simulation, and theoretical research shows that it is likely that both mechanisms each play a role in different regions of the plasma and at different operating conditions. Fortunately, although a complete understanding of the electron dynamics has so far proven to be elusive, it is known that the electron dynamics can be influenced by controlling the shape and strength of the magnetic field. At least in this sense, it is fair to say then that the magnetic field is the most important aspect of Hall thruster design.

The significance of the magnetic field in achieving efficient operation at high-specific impulse has been verified with the NASA-173M Hall thrusters. Experiments with the first thruster, the NASA-173M version 1 , validated the plasma lens design by showing how altering the magnetic field topography at high-specific impulse enhanced efficiency. ${ }^{16}$ In part 1 of this paper, ${ }^{22}$ experiments with the second thruster, the NASA-173M version 2 (v2), are reported that demonstrate there is a minimum current density and optimum magnetic field topography at which efficiency monotonically increases with voltage. Comparison of the thrusters has also shown that efficiency can be optimized for specific impulse by varying the concavity of the plasma lens. ${ }^{22}$

Design and experiment have established that Hall thrusters can operate efficiently at high-specific impulse. However, a need still exists to improve our understanding of the relationship between the thruster design and the plasma to further increase the performance, thermal margin, stability, and lifetime of future designs. To that end, a series of diagnostics were deployed with the NASA$173 \mathrm{Mv} 2$ to study the competing mechanisms affecting performance at high-specific impulse. ${ }^{18-21}$

In this paper, we consider the question of how the magnetic field enabled high-efficiency operation of the NASA-173Mv2. We begin by presenting the relevant details of a performance model derived in Ref. 21. Select results from the diagnostic studies are then reviewed and analyzed in the context of the model. Our analysis demonstrates how efficient, high-specific impulse operation of the NASA-173Mv2 was enabled through the regulation of the electron current with the magnetic field. Furthermore, we show that efficient operation was achieved over a limited range of electron Hall parameters by showing that the Hall parameter was nearly constant over a broad range of discharge voltages.

\section{Phenomenological Model of Hall Thruster Efficiency}

In this section, the essential details from a phenomenological Hall thruster performance model are presented. ${ }^{21}$ The model expresses the performance of a Hall thruster as a function of the utilization efficiencies of current, mass, voltage, and charge by accounting for a partially ionized plasma consisting of multiply charged ions. When combined with experimental data, the model can be used to weigh the relative importance of various plasma properties affecting performance such as multiply charged ions or the electron current. Although the model can be used to compute each of the performance parameters, only those portions of the model concerning efficiency are presented here.

Excluding the electrical efficiency of the systems that deliver power to a Hall thruster, the total thrust efficiency $\eta_{t}$ is the ratio of jet power in the exhaust to the total input power

$$
\eta_{t}=P_{\mathrm{jet}} / P_{t}=\left(T^{2} / 2 \dot{m}_{a} P_{d}\right)\left(P_{d} / P_{t}\right)\left(\dot{m}_{a} / \dot{m}_{t}\right)=\eta_{a} \eta_{\mathrm{mag}} \eta_{c}
$$

The cathode efficiency $\eta_{c}$ and electromagnet efficiency $\eta_{\text {mag }}$ account for the cathode flow rate and the power supplied to the electromagnet coils, respectively. Because these losses are not directly related to the production of useful thrust, the focus here is on the anode efficiency $\eta_{a}$.

The anode efficiency is determined by the efficiency of the ionization and acceleration processes. Although these processes are interrelated and not easily separated analytically, one way to decompose the anode efficiency is by defining the following utilization efficiencies.

1) Charge utilization efficiency is the net efficiency decrease due to multiply charged ions.

2) Voltage utilization efficiency is the conversion of voltage into axially directed ion velocity.

3) Current utilization efficiency is the fraction of ion current contained in the discharge current.

4) Mass utilization efficiency is the conversion of neutral mass flux into ion mass flux.

The anode efficiency is then the product of these four processes given by

$$
\eta_{a}=T^{2} / 2 \dot{m}_{a} P_{d}=\eta_{q} \eta_{v} \eta_{b} \eta_{m}
$$

where the partial efficiencies are the charge utilization efficiency

$$
\eta_{q}=\frac{\left(\sum \Omega_{i} / \sqrt{Z_{i}}\right)^{2}}{\sum \Omega_{i} / Z_{i}}
$$


the voltage utilization efficiency

$$
\eta_{v}=V_{a} / V_{d}=1-V_{l} / V_{d}
$$

the current utilization efficiency

$$
\eta_{b}=I_{b} / I_{d}=1-\varepsilon
$$

and the mass utilization efficiency

$$
\eta_{m}=\frac{\dot{m}_{b}}{\dot{m}_{a}}=\xi \eta_{b} \sum \frac{\Omega_{i}}{Z_{i}}
$$

(Throughout this paper, summations are for each ion species $i$ from 1 to $N$, where $N$ is the total number of ion species.) The current, mass, and charge utilization efficiencies are interrelated due to their dependence on the ion current and the ion current fractions. Thus, the anode efficiency can also be expressed as

$$
\eta_{a}=\underbrace{\left(1-\frac{V_{l}}{V_{d}}\right)}_{\eta_{v}} \underbrace{(1-\varepsilon)^{2} \xi\left(\sum \frac{\Omega_{i}}{\sqrt{Z_{i}}}\right)^{2}}_{\eta_{b} \eta_{m} \eta_{q}}
$$

The electron current fraction $\varepsilon$ can be calculated using Eq. (9) if the anode efficiency, ion current fractions, and ion loss voltage are known. Equations (5-8) can then be used to compute each of the utilization efficiencies. For the experiments reported here, the anode efficiency was measured with a thrust stand, the ion current fractions were measured with an ExB probe, and the ion loss voltage was measured with a retarding potential analyzer.

\section{Experimental Apparatus}

\section{A. Hall Thruster}

Shown as Fig. 1 in part 1 of this paper (Ref. 22) the laboratorymodel NASA-173Mv2 is a single-stage, magnetic layer Hall thruster sized for a nominal discharge current of $10 \mathrm{~A}$ and discharge voltages of 500-800 V. The discharge chamber has an outer diameter of $173 \mathrm{~mm}$ and is made from boron nitride. The magnetic circuit is similar to the generic Hall thruster schematic shown as Fig. 3 in Ref. 22. A fixed structure of iron pole pieces, an inner coil and an outer coil, form the primary magnetic circuit. Fine control of the magnetic field is provided with an internal trim coil and an external trim coil. The internal trim coil primarily affects the radial magnetic field in the discharge chamber, whereas the external trim coil affects the magnetic field downstream of the exit plane and near the cathode.

The thruster was powered with commercially available power supplies. A $100-\mu \mathrm{F}$ capacitor in parallel with the main discharge supply was used as the discharge filter. A laboratory-model hollow cathode rated for emission currents up to $20 \mathrm{~A}$ was used to supply electrons to the discharge chamber for ionization and the plume for neutralization. The cathode heater and keeper were used only during the thruster ignition sequence. Xenon ( $99.9995 \%$ pure) was supplied with commercially available mass flow controllers that were calibrated before each experiment using a constant-volume method. The uncertainty of the mass flow calibrations were on average $\pm 1.0 \%$. Thruster telemetry was acquired using a 22-bit datalogger that was calibrated using digital multimeters with uncertainties of $\pm 0.05 \%$ for voltage and $\pm 0.2 \%$ for current.

\section{B. Vacuum Facility}

All experiments were conducted in Vacuum Facility 12 (VF12) at NASA John H. Glenn Research Center. ${ }^{22}$ VF12 is a cryogenically pumped, cylindrical, stainless steel vacuum chamber that is $3 \mathrm{~m}$ in diameter and $9.6 \mathrm{~m}$ in length. The NASA-173Mv2 was always mounted on a thrust stand, with the thruster centerline near the chamber's vertical centerline, and fired $8.9 \mathrm{~m}$ down the length of the tank toward the cryopanels, which are located along the back-half of VF12. When the plume probes were used (Secs. III.D and III.E), they were located $2 \mathrm{~m}$ downstream of the thruster exit plane on thruster centerline, within an accuracy of $\pm 0.5 \mathrm{~cm}$ in both the axial and radial directions. (Only one probe was in VF12 at a time during testing.) A nitrogen calibrated hot-cathode ionization gauge, which was located $5.2 \mathrm{~m}$ downstream of the thruster, recorded the background pressure. The indicated pressure measurements were corrected for xenon using the base pressure on air (typically $1.0 \times 10^{-7}$ torr) and a correction factor of 2.87 for xenon. ${ }^{21}$ For a total xenon flow rate of $11 \mathrm{mg} / \mathrm{s}$, the corrected pressure was $4.6 \times 10^{-6}$ torr.

\section{Thrust Stand}

Thrust measurements were taken using a null-mode, invertedpendulum thrust stand that had been used previously in VF12 with a $10-\mathrm{kW}$ Hall thruster. ${ }^{31}$ The thrust stand was described in detail in Ref. 22. Measurement uncertainty was predominantly due to thermal drift and was estimated to be $\pm 0.5 \mathrm{mN}$. Repeatability was quantified by periodically returning to select operating points and was influenced more by the thruster, for example, by returning to the same discharge current, than the thrust stand. For a given operating point, the average repeatability of thrust measurements was $\pm 1 \%$ of the reported values. Given the uncertainty of the thrust, mass flow rate, current, and voltage, the propagated uncertainty of the efficiency was $\pm 2.3 \%$. For example, if the measured efficiency was 0.500 , then the absolute uncertainty would be \pm 0.012 .

\section{ExB Probe}

An ExB probe, or Wien filter, is a bandpass ion filter that selects ions according to their velocities through the application of crossed electric and magnetic fields. ${ }^{32-35}$ Most probes establish a constant magnetic field with permanent magnets while the electric field is established between two parallel plates. Sweeping the plate voltage while monitoring the ion current that passes through the probe yields a current-voltage characteristic that is related to the ion velocity distribution function. Because the velocity of multiply charged ions in Hall thrusters is proportional to the square root of their charge state, an ExB probe can be used to discriminate between ion species. Analysis of the ion current peaks from the probe characteristic can then be used to compute the ion species fractions.

The ExB probe used in these experiments was based on previous designs used to study ion and Hall thrusters ${ }^{33-35}$ and was described in detail in Ref. 21. The acceptance angle of the probe was $0.7 \mathrm{deg}$. Probe resolution, based on equations from Ref. 33, was conservatively estimated as $7 \%$ of the ion energy. The entrance and exit collimators were aligned perpendicular to the thruster exit plane within an accuracy of $\pm 0.5 \mathrm{deg}$. After considering the known sources of error, ${ }^{21}$ the absolute measurement uncertainty of the species fractions was estimated to be $\pm 0.04, \pm 0.02$, and \pm 0.01 for $\mathrm{Xe}^{+}, \mathrm{Xe}^{2+}$, and $\mathrm{Xe}^{3+}$, respectively. At $300 \mathrm{~V}$, this equated to a percentage uncertainty of $\pm 4 \%, \pm 50 \%$, and $\pm 100 \%$ for the species fractions of $\mathrm{Xe}^{+}, \mathrm{Xe}^{2+}$, and $\mathrm{Xe}^{3+}$, respectively. These uncertainties include the effects of using a single, axial sampling location during the measurements as inputs to the performance model. A more accurate method would include the radial variation of the species fractions, weighted by the local current density, so that a global average for the thruster could be computed. Because the experiments were limited to axial measurements, the additional uncertainty due to neglecting radial variations were instead included in the uncertainty estimates.

\section{E. Retarding Potential Analyzer}

A retarding potential analyzer (RPA) selectively filters ions by applying a retarding potential across an inlet grid. ${ }^{36,37}$ The probe acts as a high-pass filter by allowing only ions with voltages, that is, energy-to-charge ratios, greater than the grid voltage to pass and reach a collection electrode. The derivative of the resulting current-voltage characteristic is then proportional to the ion voltage distribution function. ${ }^{36}$

The RPA used in this investigation was a three-grid design described in detail in Ref. 21. Measurements of the ion voltage distribution were taken with respect to facility ground. Plasma potential $V_{p}$ measurements taken with a cylindrical langmuir probe (not shown, see Ref. 21) were used to correct the RPA data so that the true ion voltage distribution could be computed, that is, $V_{\text {true }}=V_{\text {rpa }}-V_{p}$. The most-probable ion voltage $V_{\mathrm{mp}}$ and the ion loss voltage $V_{l}$ were 
then found from the true ion voltage distribution. The most-probable ion voltage was defined as the voltage where the ion current was greatest. The ion loss voltage was then computed as the difference between the discharge voltage and the most-probable ion voltage, that is, $V_{l}=V_{d}-V_{\mathrm{mp}}$. The acceptance angle of the probe was $45 \mathrm{deg}$. The face of the probe inlet was aligned parallel to the thruster exit plane within an accuracy of $\pm 0.5 \mathrm{deg}$. After considering the known sources of error, ${ }^{21}$ the uncertainty of the most-probable voltage was estimated as $+10 /-20 \mathrm{~V}$. Similar to the ExB probe described earlier, these uncertainties include the effects of using a single, axial sampling location during the measurements as inputs to the performance model.

\section{F. Hall Probe}

A commercially available, three-axis Hall probe was used to measure the radial magnetic field of the thruster at atmospheric conditions (no plasma). The Hall probe, which was described in detail in Ref. 21, was placed on the channel centerline at the axial location of the maximum radial magnetic field, $B_{r, \max }$, which is near the discharge chamber exit plane. Before collection of data, the Hall probe was zeroed using a zero-Gauss chamber with the thruster coils off and the probe located $300 \mathrm{~mm}$ from the thruster. Measurement uncertainty, which was primarily due to positional and alignment errors, was estimated as $\pm 10 \%$.

\section{Results}

Performance and probe measurements of the thruster were conducted at xenon mass flow rates of $10 \mathrm{mg} / \mathrm{s}$ through the anode and $1.0 \mathrm{mg} / \mathrm{s}$ through the cathode. These flow rates have proven to be an acceptable balance between performance, thermal margin, and stability up to discharge voltages of $1000 \mathrm{~V}$ (Ref. 21).

The performance evaluation discussed in part 1 (Ref. 22) established the coil currents that maximized thruster efficiency. The general method for taking data was to set the voltage and flow rate and then investigate the effects of each coil on discharge current, plasma oscillations, and anode efficiency. The effects of changing the magnetic field topography were investigated by energizing the thruster coils in different combinations, which are labeled subsequently as 1) inner coil-outer coil, only the inner coil and outer coil were energized; 2) internal trim coil — the internal trim coil was energized in addition to the inner coil and outer coil; 3) internal trim coil, external trim coil-both trim coils were energized in addition to the inner coil and outer coil; and 4) external trim coil—the external trim coil was energized in addition to the inner coil and outer coil.

The probe measurements used the coil currents found during the performance evaluation that maximized efficiency. The performance measurements typically did not include data with the external trim coil unless the internal trim coil was also energized. During the ExB probe and RPA experiments, the external trim coil combination was included at all discharge voltages so that the effects of the external trim coil could be investigated separate from the internal trim coil.

For all of the experiments, the thruster was operated for $2-4 \mathrm{~h}$ after initial exposure to vacuum conditions to allow for outgassing of the discharge chamber walls. On subsequent thruster shutdowns and restarts or a change in the discharge voltage, the thruster was operated for at least 30-60 min before data were acquired. This procedure allowed enough time for the discharge current to reach a steady-state value.

\section{A. Anode Efficiency}

Anode efficiency measurements using the thrust stand were taken during the performance characterization discussed in part 1(Ref. 22) of this paper and are tabulated in Ref. 21. Figure 1 here shows that the anode efficiency at $10 \mathrm{mg} / \mathrm{s}$ increased monotonically with discharge voltage from $56.5 \%$ at $300 \mathrm{~V}$ to $68.5 \%$ at $1000 \mathrm{~V}$. Total efficiency (not shown) spanned the range from $51.1 \%$ at $300 \mathrm{~V}$ to $60.8 \%$ at $1000 \mathrm{~V}$. While providing a modest improvement to performance, trim coils were not required to obtain a continuously increasing efficiency-specific impulse characteristic. This was one indication that the magnetic circuit of the thruster was optimized for highspecific impulse operation. Additional details on the effects of the

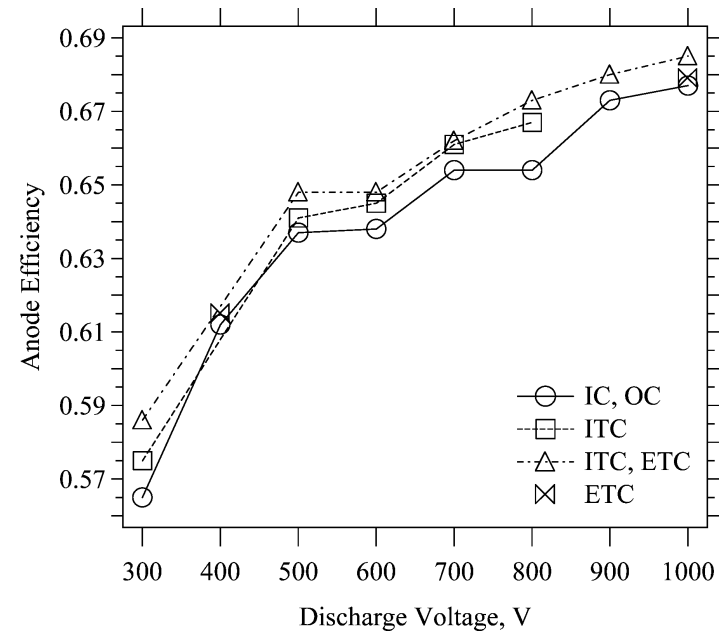

Fig. 1 Anode efficiency vs discharge voltage at $10 \mathrm{mg} / \mathrm{s}$.

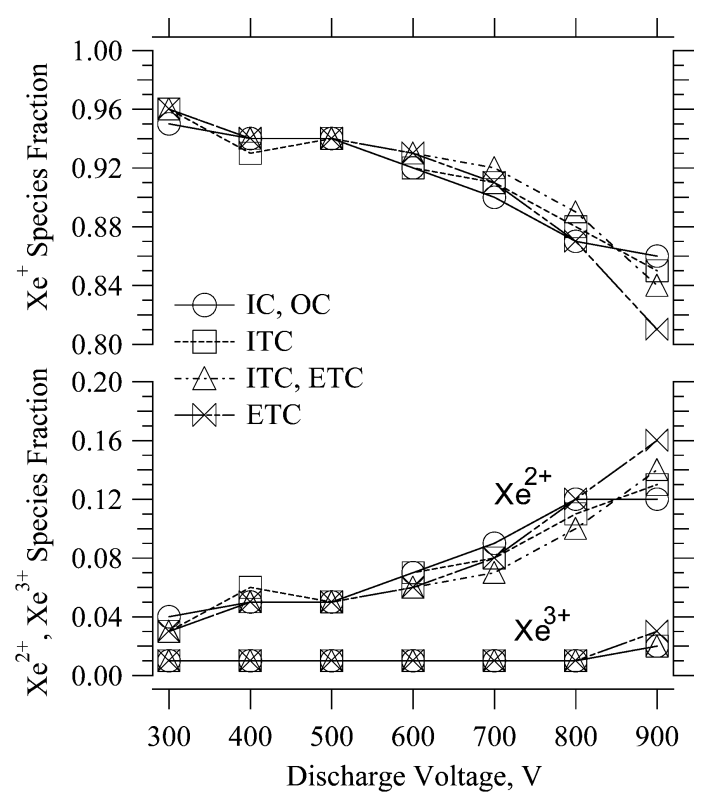

Fig. 2 Ion species fractions vs discharge voltage at $10 \mathrm{mg} / \mathrm{s}$.

applied magnetic field on thruster characteristics are discussed in Ref. 22.

\section{B. Ion Species Fractions}

ExB probe measurements of the ion species fractions were taken at discharge voltages between 300 and $900 \mathrm{~V}$ (Ref. 21). The ion species fractions are shown in Fig. 2 as a function of discharge voltage and magnetic field configuration. Although the fraction of multiply charged ions increased with voltage, $\mathrm{Xe}^{+}$still dominated the plasma at all voltages. At constant discharge voltage, there was no systematic dependence of the species fractions with the magnetic field configuration. The 7\% energy resolution of the ExB probe may have contributed to this result by masking small changes of the ion species fractions. Between 300 and $900 \mathrm{~V}$, the species fraction of $\mathrm{Xe}^{+}$decreased from 0.96 to 0.81 , whereas the $\mathrm{Xe}^{2+}$ species fraction increased from 0.03 to 0.16 and the $\mathrm{Xe}^{3+}$ species fraction increased from 0.01 to 0.03 . At $300 \mathrm{~V}$, the fractions of $\mathrm{Xe}^{2+}$ and $\mathrm{Xe}^{3+}$ were lower, but still consistent with, Hall thruster studies that have reported species fractions of 0.06-0.11 $\mathrm{Xe}^{2+}$ and $0.01 \mathrm{Xe}^{3+}$ (Refs. 35, 38 , and 39).

\section{Ion Loss Voltage}

RPA measurements of the ion loss voltage were taken at discharge voltages between 300 and $800 \mathrm{~V}$ (Ref. 21). A grid short inside the RPA ended testing at $800 \mathrm{~V}$ after testing only with the inner coil and outer coil. The ion loss voltage is shown in Fig. 3 as a function 


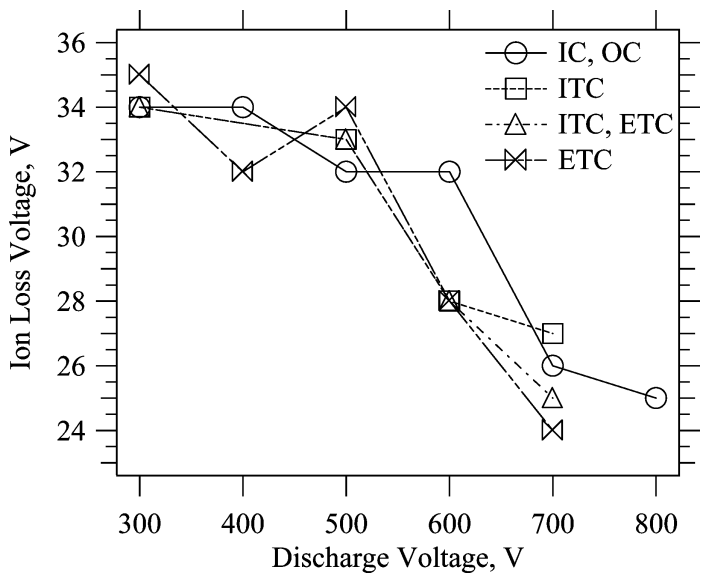

Fig. 3 Ion loss voltage vs discharge voltage at $10 \mathrm{mg} / \mathrm{s}$.

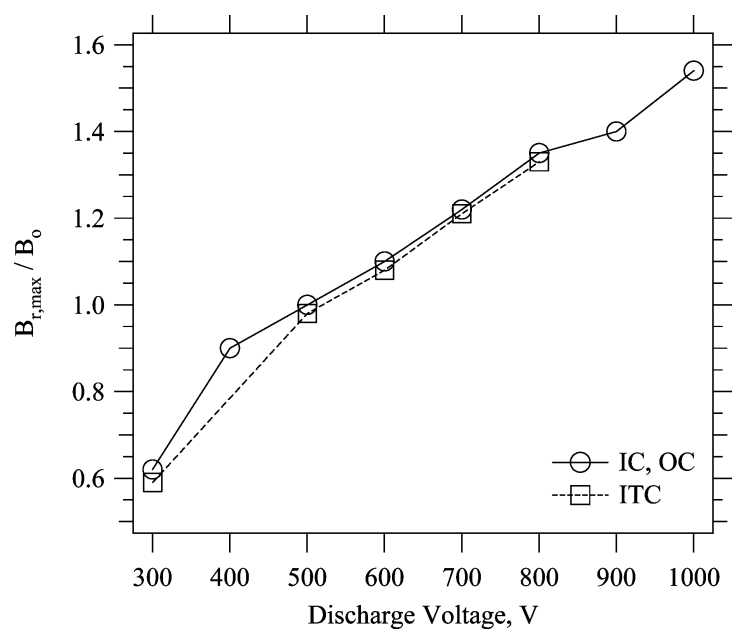

Fig. 4 Radial magnetic field vs discharge voltage at $10 \mathrm{mg} / \mathrm{s}$.

of discharge voltage and magnetic field configuration. At constant discharge voltage, there was no systematic dependence of the ion loss voltage with the magnetic field. As in the case of the ExB probe, this trend may have been due to the limited voltage resolution of the RPA. The ion loss voltage decreased with discharge voltage from 35 $\mathrm{V}$ at $300 \mathrm{~V}$ to $25 \mathrm{~V}$ at $800 \mathrm{~V}$. At $300 \mathrm{~V}$, the ion loss voltage was lower, but still consistent with Hall thruster studies that have reported ion loss voltages between 45 and $50 \mathrm{~V}$ (Refs. 35, 39, and 40).

\section{Magnetic Field Strength}

Measurements with the Hall probe of the maximum, radial magnetic field strength on the channel centerline were taken at the coil combinations corresponding to discharge voltages of 300-1000 V (Ref. 21). The magnetic field strength, normalized by an arbitrary constant $B_{0}$, is shown in Fig. 4 for each of the coil combinations from the performance characterization. Operating points where the external trim coil was energized are not explicitly shown because the external trim coil has a negligible effect on the magnetic field in the discharge chamber. The magnetic field increase was nearly linear with voltage. Between 300 and $400 \mathrm{~V}$, the magnetic field abruptly jumped and then linearly increased between 400 and $1000 \mathrm{~V}$. The increase in magnetic field between 300 and $400 \mathrm{~V}$ corresponded to increased plume divergence and discharge current oscillations also measured between 300 and $400 \mathrm{~V}$ (not shown, see Ref. 22). The magnetic field increased by a factor of 1.5 between 300 and $400 \mathrm{~V}$ and by a factor of 1.7 between 400 and $1000 \mathrm{~V}$. Between 300 and $1000 \mathrm{~V}$, the magnetic field increased by a factor of 2.6.

\section{Discussion}

\section{A. Charge Utilization Efficiency}

Figure 5 shows the charge utilization efficiency computed with Eq. (5). In Fig. 5 and as shown subsequently, error bars representing

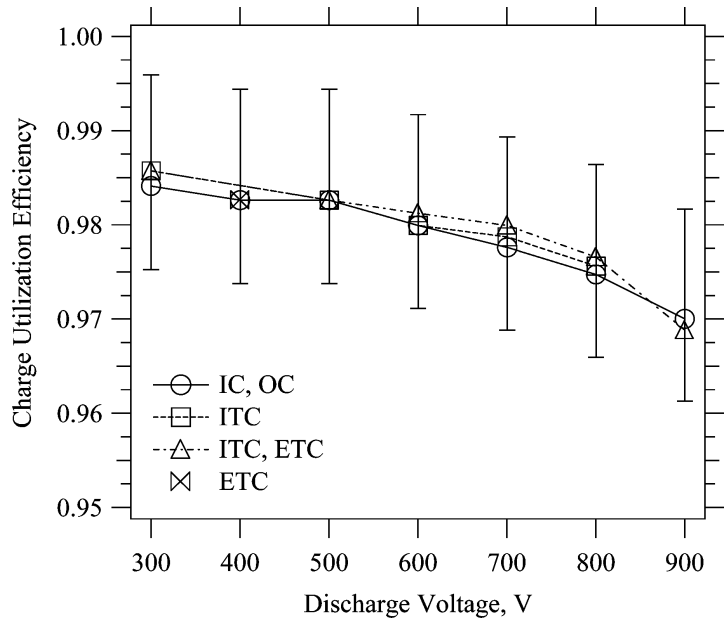

Fig. 5 Charge utilization efficiency vs discharge voltage at $10 \mathrm{mg} / \mathrm{s}$ (compressed scale on $y$ axis).

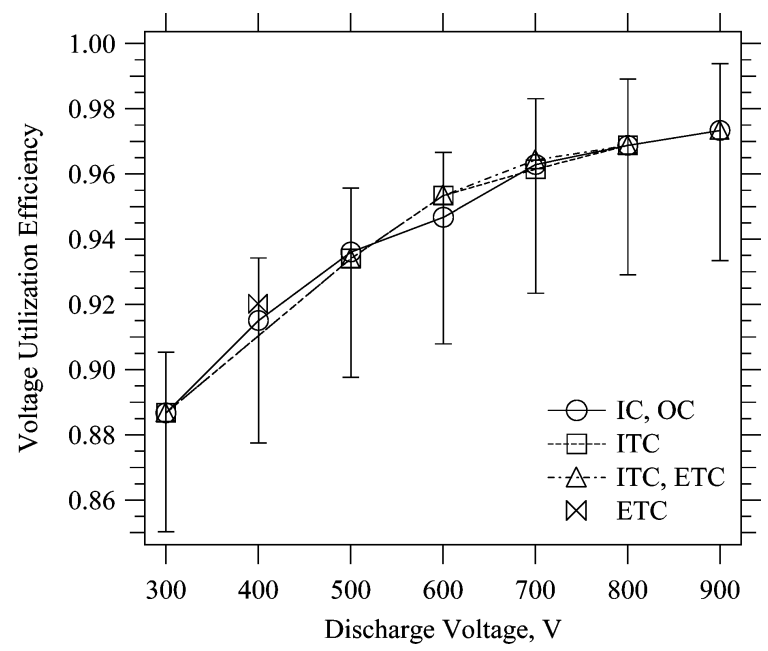

Fig. 6 Voltage utilization efficiency vs discharge voltage at $10 \mathrm{mg} / \mathrm{s}$; data at 800-900 V extrapolated except for $800 \mathrm{~V}$, IC, OC point.

the propagated uncertainty are shown on the inner coil, outer coil coil combination only. The charge utilization averaged $98.5 \%$ at 300 $\mathrm{V}$ and then decreased at a nearly linear rate to an average of $97.6 \%$ at $800 \mathrm{~V}$. A (relatively) sharp decrease occurred between 800 and $900 \mathrm{~V}$, where the charge utilization dropped to an average of $97.0 \%$.

Although the charge utilization defined by Eq. (5) does not include the effects of multiply charged ions on the current or mass utilization, the charge utilization is a measure of the net effect of multiply charged ions on performance. ${ }^{21}$ Thus, the net decrease of the anode efficiency due to multiply charged ions averaged $1.5-3.0 \%$ between discharge voltages of 300 and $900 \mathrm{~V}$. This statement is not meant to imply that multiply charged ions can be neglected in our analysis. Multiply charged ions significantly affect the ion current calculation and must be included (Sec. V.C).

\section{B. Voltage Utilization Efficiency}

Figure 6 shows the voltage utilization efficiency computed with Eq. (6). The voltage utilization was computed only for the thruster operating points, that is, coil combinations, that were studied during the performance characterization. The RPA data include all of the thruster operating points from the performance characterization between 300 and $700 \mathrm{~V}$. At $800 \mathrm{~V}$, data with the RPA were acquired only with the inner coil and outer coil, and no data were collected with the RPA at $900 \mathrm{~V}$. Because the voltage utilization showed a clear trend with discharge voltage, the ion loss voltage was conservatively extrapolated for the missing data points at 800 and $900 \mathrm{~V}$. The ion loss voltage was set to $25 \mathrm{~V}$ for the operating conditions at 


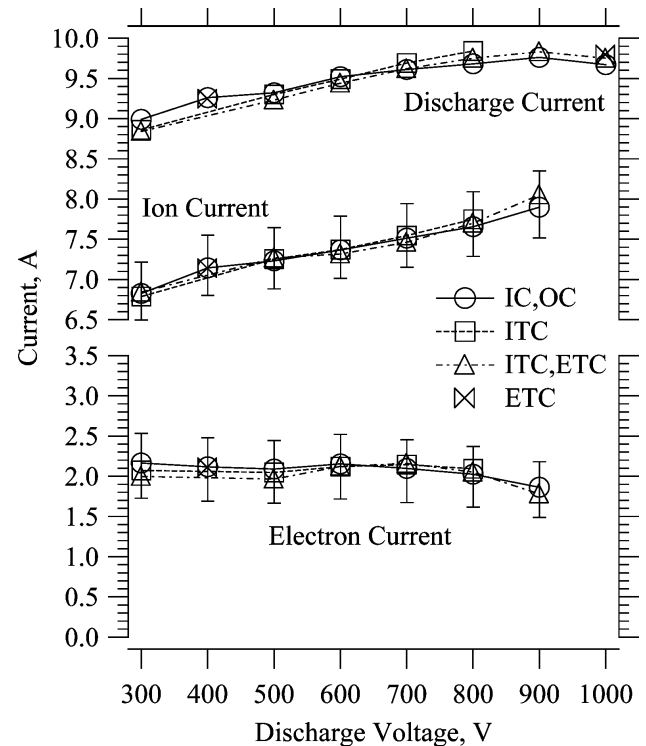

Fig. 7 Discharge, ion, and electron current vs discharge voltage at $10 \mathrm{mg} / \mathrm{s}$.

$800 \mathrm{~V}$ using the trim coils (the same as the measured value at 800 $\mathrm{V}, \mathrm{IC}$ and OC). The ion loss voltage was set to $24 \mathrm{~V}$ for each data point at $900 \mathrm{~V}$, which was consistent with the observed decrease in ion loss voltage with increasing discharge voltage. Because Eq. (9) depends on the ion loss voltage, the computations that follow in Secs. V.C-V.G are also based on these extrapolations.

Figure 6 shows that the voltage utilization averaged $88.7 \%$ at $300 \mathrm{~V}$ and increased to an average of $96.9 \%$ at $800 \mathrm{~V}$. The voltage utilization was $97.3 \%$ for the extrapolated data at $900 \mathrm{~V}$. The increase of the voltage utilization was primarily the result of the increasing discharge voltage because the decrease of the ion loss voltage was negligible in comparison.

\section{Electron and Ion Current}

Figure 7 shows the discharge current and the ion and electron current computed with Eq. (9). The electron current was relatively constant between 300 and $700 \mathrm{~V}$ and then decreased between 700 and $900 \mathrm{~V}$. The average value of the electron current between 300 and $900 \mathrm{~V}$ was $2.06 \mathrm{~A}$ with a maximum deviation of $+5 \% /-14 \%$ from the average. In contrast, the ion current steadily increased with discharge voltage from a minimum of $6.79 \mathrm{~A}$ at $300 \mathrm{~V}$ to a maximum of $8.05 \mathrm{~A}$ at $900 \mathrm{~V}$. The same relative increase of the ion current with voltage has been confirmed with unpublished results on the same thruster from a cathode-plane Faraday probe that employed a method similar to that found in Ref. 18. The discharge current also increased with discharge voltage from a minimum of $8.84 \mathrm{~A}$ at $300 \mathrm{~V}$ to a maximum of $9.83 \mathrm{~A}$ at $900 \mathrm{~V}$. Thus, the increase in the discharge current was attributed to the increasing fraction of multiply charged ions, rather than an increase of electron current. This demonstrates that the electron current in a high-specific impulse Hall thruster can be regulated if the magnetic field is of the appropriate shape and strength. These results also show that an increasing discharge current characteristic should not be assumed to be the sole result of increasing electron current, especially if the thruster is operating efficiently. Finally, these results underscore the importance of including the effects of multiply charged ions when analyzing the efficiency of high-specific impulse Hall thrusters.

\section{Current Utilization Efficiency}

Figure 8 shows the current utilization efficiency computed with Eq. (7) by using the ion and electron currents calculated in Sec. V.C. The continuous increase of the ion current with voltage shown in Fig. 7 was not reflected in the current utilization because the current utilization depends on the ion and electron current. Instead, the current utilization initially increased between 300 and $500 \mathrm{~V}$, was

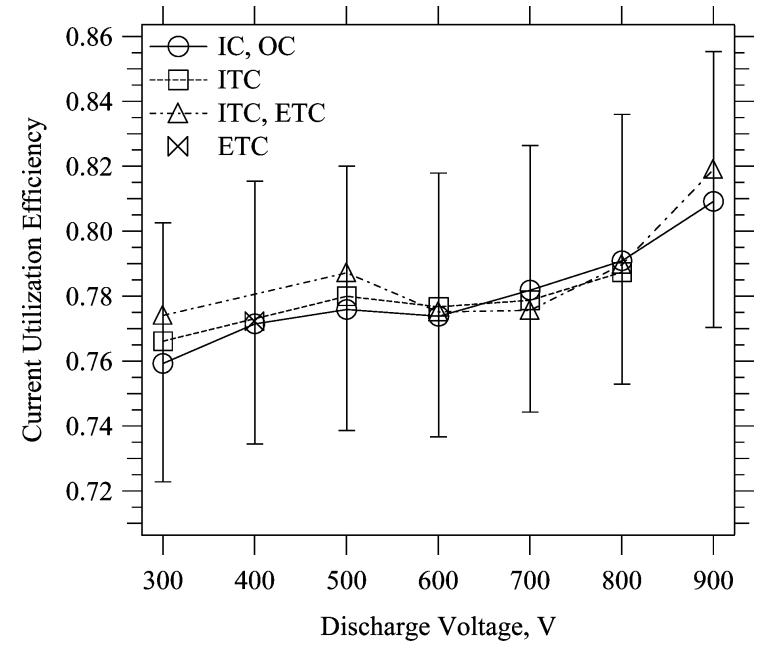

Fig. 8 Current utilization efficiency vs discharge voltage at $10 \mathrm{mg} / \mathrm{s}$.

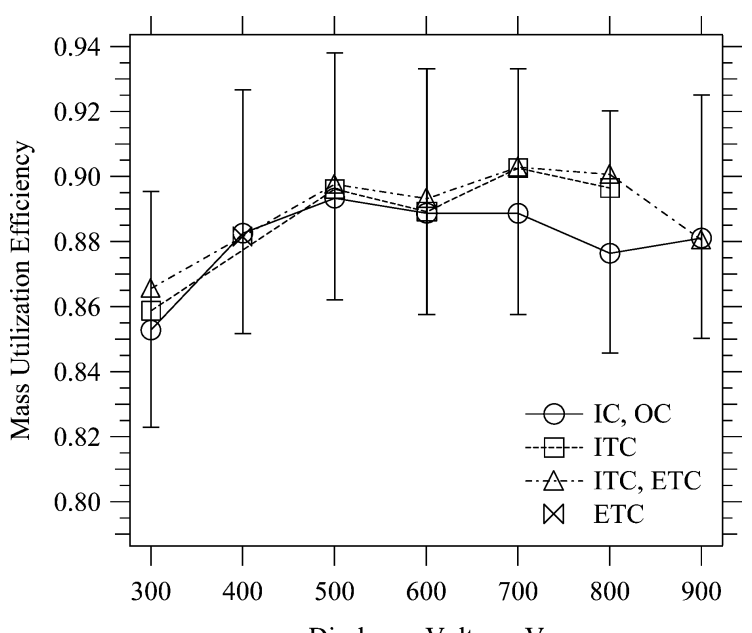

Fig. 9 Mass utilization efficiency vs discharge voltage at $10 \mathrm{mg} / \mathrm{s}$.

relatively constant between 500 and $700 \mathrm{~V}$, and then increased between 700 and $900 \mathrm{~V}$. The rise in the current utilization beginning at $700 \mathrm{~V}$ was largely the result of the electron current decreasing. Overall, because the electron current was nearly constant between 300 and $900 \mathrm{~V}$, the current utilization efficiency increased with voltage because of the increasing fraction of multiply charged ions. The current utilization averaged $76.6 \%$ at $300 \mathrm{~V}$ and increased to an average of $81.4 \%$ at $900 \mathrm{~V}$. The computed current utilization efficiencies were consistent with values typically found in the literature for Hall thrusters, ${ }^{41}$ as well as with the value of $77 \%$ previously measured using a cathode-plane Faraday probe with the thruster operating at $500 \mathrm{~V}, 5 \mathrm{mg} / \mathrm{s}$ in Ref. 18.

\section{E. Mass Utilization Efficiency}

Figure 9 shows the mass utilization efficiency computed with Eq. (8) by using the ion current that was calculated in Sec. V.C. The mass utilization increased between 300 and $500 \mathrm{~V}$, was relatively constant between 500 and $700 \mathrm{~V}$, and then decreased between 700 and $900 \mathrm{~V}$. The mass utilization averaged $85.9 \%$ at $300 \mathrm{~V}$ and $88.1 \%$ at $900 \mathrm{~V}$. The maximum mass utilization of $90.3 \%$ occurred at $700 \mathrm{~V}$.

The effects of multiply charged ions are usually neglected in the literature, resulting in mass utilization efficiencies of $90-95 \%$ for discharge voltages of $300 \mathrm{~V}$ (Refs. 41 and 42). This is an adequate approximation so long as the discharge voltage is not much greater than $300 \mathrm{~V}$. If multiply charged ions had been neglected in the calculations, the mass utilization shown in Fig. 9 would have been $3.4 \%$ (absolute) higher at $300 \mathrm{~V}$ and $9.3 \%$ (absolute) higher at $900 \mathrm{~V}$. Conversely, the current utilization shown in Fig. 8 would have then 


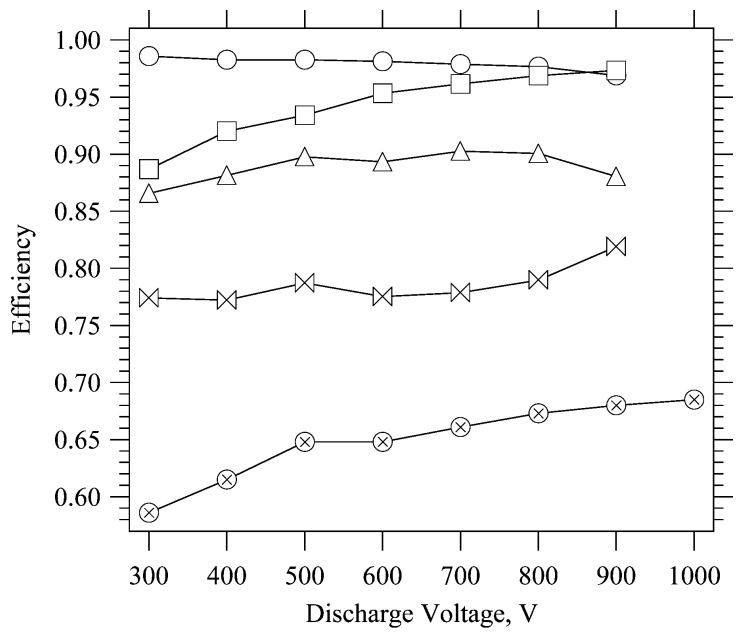

Fig. 10 Optimized anode efficiency and corresponding utilization efficiencies vs discharge voltage at $10 \mathrm{mg} / \mathrm{s}: \bigcirc$, charge; $\square$, voltage; $\triangle$, mass; $\bowtie$, current; and $\otimes$, anode. (Error bars suppressed for clarity.)

been $3.4 \%$ (absolute) lower at $300 \mathrm{~V}$ and $9.3 \%$ (absolute) lower at $900 \mathrm{~V}$. These results demonstrate that the approximation of a singly charged plasma breaks down at high voltage as the fraction of multiply charged ions increases with the discharge voltage.

\section{F. Optimized Anode Efficiency}

Figure 10 shows the anode efficiency and the corresponding utilization efficiencies for the coil combinations at each discharge voltage shown in Fig. 1 that maximized anode efficiency. Over discharge voltages ranging from 300 to $900 \mathrm{~V}$, the voltage and current utilization increased, the charge utilization decreased, and the mass utilization was nearly constant. The current utilization was always less than the other partial efficiencies, which confirmed the well-known result that Hall thruster efficiency is largely determined by the current utilization, further emphasizing the importance of the magnetic field as a means to control the electron dynamics and maximize thruster efficiency.

Because the current utilization increased with the discharge voltage (and the electron current showed evidence of a decrease above $700 \mathrm{~V}$ ), these results imply that the maximum efficiency of Hall thrusters may not have yet been reached. Anode efficiencies can potentially still be increased, perhaps as high as the $80 \%$ value reported in Ref. 43, if the discharge voltage is increased beyond $1000 \mathrm{~V}$. At discharge voltages less than $1000 \mathrm{~V}$, because the electron current was relatively constant, further efficiency improvements require a means to minimize the production of multiply charged ions while increasing the voltage and mass utilization. Because substantial increases in the charge or voltage utilization seem unlikely (because these quantities already have high values), increasing the anode efficiency below $1000 \mathrm{~V}$ will depend on a means to increase the mass utilization, such as the well-known effects of high-current density or high-power operation. ${ }^{41,42}$

\section{G. Electron Hall Parameter}

The scaling of the axial electron current with voltage provides insight on the electron Hall parameter. The Hall parameter can be computed from the ratio of the azimuthal and axial electron current densities [Eq. (1)]. The azimuthal electron current density can be calculated from

$$
j_{e \theta}=n_{e} e v_{\mathrm{ExB}}=n_{e} e\left(E_{z} / B_{r}\right)
$$

The axial electron current density can be computed from the electron current and the annular cross-sectional area of the discharge chamber given by

$$
j_{e z}=I_{e} / A
$$

After the quasi-neutrality condition $\left(n_{e} \approx n_{b}\right)$ is applied and Eqs. (10) and (11) are substituted, the Hall parameter from Eq. (1) becomes

$$
\Omega_{e}=n_{b} e E_{z} A / B_{r} I_{e}
$$

Besides the electron current and discharge chamber area, the Hall parameter was calculated from the available data as follows:

1) The axial electric field at $300 \mathrm{~V}$ was estimated from typical electric field strengths in modern Hall thrusters and then linearly varied with discharge voltage. ${ }^{18,21,44}$

2) The radial magnetic field was taken from the Hall probe measurements shown in Fig. 4.

3) The beam density $n_{b}$ was computed from an expression derived in Ref. 21 as

$$
I_{b}=\sum I_{i}=A e^{\frac{3}{2}} n_{b} \sqrt{\frac{2 V_{a}}{m_{x e}}} \sum \xi_{i} Z_{i}^{\frac{3}{2}}
$$

Because Eq. (12) is calculated based on global thruster properties, the result should be interpreted as a bulk, or average Hall parameter that is representative of the discharge chamber acceleration zone where the plasma density is high and the ions are being accelerated through the majority of the applied voltage. This is an important point to make because time-averaged measurements of the Hall parameter from Refs. 45-47 have shown a strong spatial dependence throughout the discharge chamber. These studies have shown that the Hall parameter attains a maximum value of several hundred in the acceleration zone and then falls to the Bohm value, that is, 16, near the anode and downstream of the exit plane.

Figure 11 shows that the average Hall parameter computed with Eq. (12) was an order of magnitude larger than the Bohm value. The error bars include a conservative $25 \%$ uncertainty on the electric field used in the calculations, resulting in an overall uncertainty of $+57 /-34 \%$. This relatively large uncertainty is consistent with other probe-based measurements found in the literature (typically $50 \%$ due to their reliance on plasma densities derived from langmuir probes). At $300 \mathrm{~V}$, the Hall parameter was on average 210 . Between 300 and $400 \mathrm{~V}$, the Hall parameter decreased by $25 \%$ and then was nearly constant between 400 and $900 \mathrm{~V}$. Between 400 and $900 \mathrm{~V}$, the Hall parameter was, on average, 160 with a maximum deviation from the average of $+12 \% /-7 \%$. The computed Hall parameter was consistent with experimental investigations ${ }^{45-47}$ that have shown that the Hall parameter attains a value of several hundred in the acceleration zone and with numerical simulations ${ }^{25,30}$ that have found acceptable results when a fitting constant related to the Hall parameter was approximately 100 .

The decrease in the Hall parameter between 300 and $400 \mathrm{~V}$ likely coincided with the onset of space-charge saturation of the

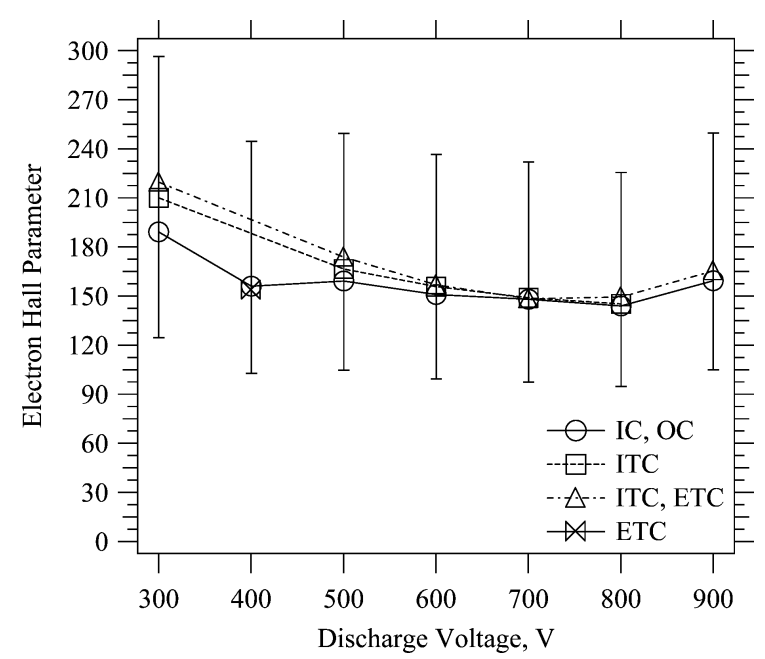

Fig. 11 Electron Hall parameter vs discharge voltage at $10 \mathrm{mg} / \mathrm{s}$. 
discharge chamber wall sheaths due to intense secondary electron emission. ${ }^{28,29}$ In other thrusters, the space-charge saturation regime has been correlated with a sudden decrease in the breathing-mode frequency, increased electron current, and maximum efficiency. ${ }^{28,29,48}$ Whereas the NASA-173Mv2 data cannot reveal whether or not space-charge saturation occurred in our experiments, there is ample evidence that the thruster was transitioning to a different operating mode at discharge voltages of $\sim 400 \mathrm{~V}$. For example, measurements in Ref. 22 of the plume divergence and discharge current oscillations both show large increases between 300 and $400 \mathrm{~V}$. Also, the magnetic field intensity (Fig. 4) also sharply increased between 300 and $400 \mathrm{~V}$. The transition to this new regime most likely persisted until about $600 \mathrm{~V}$, after which discharge current oscillations suddenly decreased, while the breathing-mode frequency (not shown, see Ref. 22) continued to increase. Whereas the precise details are uncertain and require further research, these trends suggest that the applied magnetic field was effective at controlling oscillations and wall effects such that the electron current was stabilized, thereby allowing the anode efficiency to increase continuously with discharge voltage.

At least between 400 and $1000 \mathrm{~V}$, maintaining a nearly constant Hall parameter over such a large voltage range implied that the balance of all of the processes affecting the axial electron mobility was also approximately constant. This result supports our earlier claim that efficient thruster operation can only be achieved over a limited range of Hall parameters.

\section{Conclusions}

When combined with data from performance and plasma measurements, the results from a phenomenological Hall thruster performance model have provided new insights on the factors affecting efficiency during high-specific impulse operation. Between discharge voltages of 300 and $900 \mathrm{~V}$, analysis has shown that, although the net decrease of efficiency due to multiply charged ions was at most $3.0 \%$, the effects of multiply charged ions on the discharge current and electron current could not be neglected. The increase of the discharge current with discharge voltage was attributed to the increasing fraction of multiply charged ions, whereas the electron current was found to be nearly constant. These findings revealed how efficient operation at high-specific impulse was enabled through the regulation of the electron current with the applied magnetic field. Additionally, the current utilization was always less than the other partial efficiencies composing the anode efficiency, which underscored the importance of the magnetic field as a means of controlling the electron dynamics and maximizing thruster efficiency. Finally, it was shown that the electron Hall parameter was nearly constant with voltage, which supported our claim that efficient operation can be achieved only over a narrow range of Hall parameters.

The research on the NASA-173M Hall thrusters has established a solid foundation from which the development of high-specific impulse Hall thrusters may continue. Whereas the design of such thrusters is currently in progress, there are several other issues and potential directions that deserve further scrutiny. These topics are briefly discussed next.

\section{Numerical Simulations}

Although our analysis has provided new insights not previously available, a more fundamental explanation of the factors affecting efficiency at high-specific impulse will most likely require numerical modeling. Such efforts might be similar to those of Barral et al. ${ }^{29}$ and most certainly must include multiply charged ions.

\section{Lifetime Evaluation}

This research has tacitly assumed that if thruster efficiency is maximized so too is thruster lifetime. Whereas this may prove to be accurate, there are still questions concerning the rate at which lifetime decreases with increasing voltage. Modeling by Garrigues et al. has begun to address some of these issues by demonstrating how erosion rates in 1600-s specific impulse thrusters decrease when a plasma lens is used (in lieu of less concave magnetic field lines).
Efforts to predict the operational lifetime of other Hall thrusters are also currently be pursued.

\section{Krypton Operation}

Besides offering higher specific impulse, krypton may prove to be a better propellant than xenon at high-specific impulse because the thruster lifetime should be higher with krypton. Whereas ionizing krypton requires more energy than xenon (which decreases the mass utilization efficiency), this attribute also decreases the production of multiply charged ions. The lower mass of krypton with respect to xenon also decreases the sputtering yield approximately by the square root of the mass ratio. If Hall thruster efficiency on krypton can be improved to approach that of xenon, a krypton thruster could benefit several interplanetary missions due to its higher specific impulse and longer lifetime. Recent investigations of several Hall thrusters operating on krypton have shown promise, where total efficiencies greater than $50 \%$ have been measured. ${ }^{49}$

\section{Acknowledgments}

Support for this research through the former NASA Code R Energetics program is gratefully acknowledged. We would like to thank James Haas at the U.S. Air Force Research Laboratory, Edwards Air Force Base, California for providing us with the retarding potential analyzer and participating in the experiment using the diagnostic. We would also like to thank David Jacobson, David Manzella, and Peter Peterson for technical advice and insight.

\section{References}

${ }^{1}$ Kaufman, H. R., "Technology of Closed-Drift Thrusters," AIAA Journal, Vol. 23, No. 1, 1985, pp. 78-87.

${ }^{2}$ Oleson, S. R., and Sankovic, J. M., "Advanced Hall Electric Propulsion for Future in-Space Transportation," NASA TM-210676, April 2001.

${ }^{3}$ Gulczinksi, F. S., and Spores, R. A., "Analysis of Hall-Effect Thrusters and Ion Engines for Orbit Transfer Missions," AIAA Paper 96-2973, July 1996.

${ }^{4}$ Raitses, Y., Guelman, M., Ashkenazy, J., and Appelbaum, G., "Orbit Transfer with a Variable Thrust Hall Thruster Under Drag," Journal of Spacecraft and Rockets, Vol. 36, No. 6, 1998, pp. 875-881.

${ }^{5}$ Oleson, S. R., "Advanced Electric Propulsion for Space Solar Power Satellites," NASA TM-1999-209307, Aug. 1999.

${ }^{6}$ Oleson, S. R., "Advanced Electric Propulsion for RLV Launched Geosynchronous Spacecraft,” NASA TM-1999-209646, Dec. 1999.

${ }^{7}$ Oleson, S. R., "Mission Advantages of Constant Power, Variable Isp Electrostatic Thrusters," NASA TM-2000-210477, Nov. 2000.

${ }^{8}$ Dudzinski, L. A., Hack, K. J., Gefert, L. P., Kerslake, T. W., and Hewston, A. W., "Design of a Solar Electric Propulsion Transfer Vehicle for a NonNuclear Human Mars Exploration Architecture," 26th International Electric Propulsion Conf., Paper IEPC-99-181, Oct. 1999.

${ }^{9}$ Fiehler, D., and Oleson, S., "A Comparison of Electric Propulsion Systems for Mars Exploration," NASA TM-2003-212593, Sept. 2003.

${ }^{10}$ Gefert, L. P., Hack, K. J., and Kerslake, T. W., "Options for the Human Exploration of Mars Using Solar Electric Propulsion," AIP Conf. No. 458, Space Technology and Applications International Forum (STAIF), Jan. 1999.

${ }^{11}$ Brophy, J. R., and Noca, M., "Electric Propulsion for Solar System Exploration," Journal of Propulsion and Power, Vol. 14, No. 5, 1998, pp. 700-707.

${ }^{12}$ Jacobson, D. T., Jankovsky, R. S., Rawlin, V. K., and Manzella, D. H., "High Voltage TAL Performance," NASA TM-2001-211147, Nov. 2001.

${ }^{13}$ Manzella, D. H., Jacobson, D. T., and Jankovsky, R. S., "High Voltage SPT Performance," NASA TM-2001-211135, Nov. 2001.

${ }^{14}$ Pote, B., and Tedrake, R., "Performance of a High Specific Impulse Hall Thruster," 27th International Electric Propulsion Conf., Paper IEPC-01-35, Oct. 2001

${ }^{15}$ Hofer, R. R., Peterson, P. Y., Gallimore, A. D., and Jankovsky, R. S., "A High Specific Impulse Two-Stage Hall Thruster with Plasma Lens Focusing," 27th International Electric Propulsion Conf., Paper IEPC-01-36, Oct. 2001.

${ }^{16}$ Hofer, R. R., and Gallimore, A. D., "The Role of Magnetic Field Topography in Improving the Performance of High-Voltage Hall Thrusters," AIAA Paper 2002-4111, July 2002.

${ }^{17}$ Hofer, R. R., and Jankovsky, R. S., "The Influence of Current Density and Magnetic Field Topography in Optimizing the Performance, Divergence, and Plasma Oscillations of High Specific Impulse Hall Thrusters," NASA TM-2003-212605, Dec. 2003.

${ }^{18}$ Hofer, R. R., and Gallimore, A. D., "Recent Results from Internal and Very-Near-Field Plasma Diagnostics of a High Specific Impulse Hall Thruster," NASA CR-2003-212604, Dec. 2003. 
${ }^{19}$ Hofer, R. R., and Gallimore, A. D., "Ion Species Fractions in the FarField Plume of a High-Specific Impulse Hall Thruster," NASA CR-2003212893, Dec. 2003

${ }^{20}$ Hofer, R. R., Haas, J. M., and Gallimore, A. D., "Ion Voltage Diagnostics in the Far-Field Plume of a High-Specific Impulse Hall Thruster," NASA CR-2003-212895, Dec. 2003.

${ }^{21}$ Hofer, R. R., "Development and Characterization of High-Efficiency, High-Specific Impulse Xenon Hall Thrusters," Ph.D. Dissertation, Dept. of Aerospace Engineering, Univ. of Michigan, Ann Arbor, MI, Jan. 2004; also NASA/CR-2004-213099, June 2004.

${ }^{22}$ Hofer, R. R., and Jankovsky, R. S., "High-Specific Impulse Hall Thrusters, Part 1: Influence of Current Density and Magnetic Field," Journal of Propulsion and Power (to be published).

${ }^{23}$ Janes, G. S., and Lowder, R. S., "Anomalous Electron Diffusion and Ion Acceleration in a Low-Density Plasma," Physics of Fluids, Vol. 9, No. 6, 1966.

${ }^{24}$ Morosov, A. I., Esipchuk, Y. V., Tilinin, G. N., Trofimov, A. V., Sharov, Y. A., and Shchepkin, G. Y., "Plasma Accelerator with Closed Electron Drift and Extended Acceleration Zone," Soviet Physics Technical Physics, Vol. 17, No. 1, 1972, pp. 38-45.

${ }^{25}$ Fife, J. M., "Hybrid-PIC Modeling and Electrostatic Probe Survey of Hall Thrusters," Ph.D. Dissertation, Dept. of Aeronautics and Astronautics, Massachusetts Inst. of Technology, Cambridge, MA, Sept. 1998.

${ }^{26}$ Boeuf, J. P., and Garrigues, L., "Low Frequency Oscillations in a Stationary Plasma Thruster," Journal of Applied Physics, Vol. 84, No. 7, 1998, pp. 3541-3554.

${ }^{27}$ Ivanov, A. A., Ivanov, A. A., and Bacal, M., "Effect of Plasma-Wall Recombination on the Conductivity in Hall Thrusters," Plasma Physics and Controlled Fusion, Vol. 44, No. 8, 2002, pp. 1463-1470.

${ }^{28}$ Gascon, N., Dudeck, M., and Barral, S., "Wall Material Effects in Stationary Plasma Thrusters I: Parametric Studies of an SPT-100," Physics of Plasmas, Vol. 10, No. 10, 2003, pp. 4123-4136.

${ }^{29}$ Barral, S., Makowski, K., Peradzynski, Z., Gascon, N., and Dudeck, M., "Wall Material Effects in Stationary Plasma Thrusters II: Near-Wall and in-Wall Conductivity," Physics of Plasmas, Vol. 10, No. 10, 2003, pp. 4137-4152.

${ }^{30}$ Ahedo, E., Gallardo, J. M., and Martinez-Sanchez, M., "Effects of the Radial Plasma-Wall Interaction on the Hall Thruster Discharge," Physics of Plasmas, Vol. 10, No. 8, 2003, pp. 3397-3409.

${ }^{31}$ Mason, L. S., Jankovsky, R. S., and Manzella, D. H., "1000 Hours of Testing on a 10 Kilowatt Hall Effect Thruster," AIAA Paper 2001-3773, July 2001.

${ }^{32}$ Seliger, R. L., "ExB Mass-Separator Design,” Journal of Applied Physics, Vol. 43, No. 5, 1972, pp. 2352-2357.

${ }^{33}$ Kim, S. W., "Experimental Investigations of Plasma Parameters and Species-Dependent Ion Energy Distribution in the Plasma Exhaust Plume of a Hall Thruster," Ph.D. Dissertation, Dept. of Aerospace Engineering, Univ. of Michigan, Ann Arbor, MI, 1999.

${ }^{34}$ Williams, G. J., Domonkos, M. T., and Chavez, J. M., "Measurement of Doubly Charged Ions in Ion Thruster Plumes," NASA TM-2002-211295, Jan. 2002.

${ }^{35}$ Kim, S.-W., and Gallimore, A. D., "Plume Study of a 1.35-kW SPT-100 Using an ExB Probe," Journal of Spacecraft and Rockets, Vol. 39, No. 6, 2002, pp. 904-909.

${ }^{36}$ King, L. B., "Transport-Property and Mass Spectral Measurements in the Plasma Exhaust Plume of a Hall Effect Space Propulsion System,"
Ph.D. Dissertation, Dept. of Aerospace Engineering, Univ. of Michigan, Ann Arbor, MI, 1998.

${ }^{37}$ Hutchinson, I. H., Principles of Plasma Diagnostics, 1st ed., Cambridge Univ. Press, Cambridge, England, U.K., 1987.

${ }^{38}$ King, L. B., and Gallimore, A. D., "Mass Spectral Measurements in the Plume of an SPT-100 Hall Thruster," Journal of Propulsion and Power, Vol. 16, No. 6, 2000, pp. 1086-1092.

${ }^{39}$ Gulczinksi, F. S., and Gallimore, A. D., "Near-Field Ion Energy and Species Measurements of a 5-kW Hall Thruster," Journal of Propulsion and Power, Vol. 17, No. 2, 2001, pp. 418-427.

${ }^{40}$ King, L. B., and Gallimore, A. D., "Ion-Energy Diagnostics in the Plasma Exhaust Plume of a Hall Thruster," Journal of Propulsion and Power, Vol. 16, No. 5, 2000, pp. 916-922.

${ }^{41}$ Kim, V., "Main Physical Features and Processes Determining the Performance of Stationary Plasma Thrusters," Journal of Propulsion and Power, Vol. 14, No. 5, 1998, pp. 736-743.

${ }^{42}$ Zhurin, V. V., Kaufman, H. R., and Robinson, R. S., "Physics of Closed Drift Thrusters," Plasma Sources Science and Technology, Vol. 8, No. 1, 1999, pp. R1-R20.

${ }^{43}$ Grishin, S. D., Erofeev, V. S., Zharinov, A. V., Naumkin, V. P., and Safronov, I. N., "Characteristics of a Two-Stage Ion Accelerator with an Anode Layer," Journal of Applied Mathematics and Technical Physics, Vol. 19, No. 2, 1978, pp. 166-173.

${ }^{44}$ Raitses, Y., Staack, D., Dorf, L., and Fisch, N. J., "Experimental Study of Acceleration Region in a 2 kW Hall Thruster," AIAA Paper 2003-5153, July 2003

${ }^{45}$ Haas, J. M., "Low-Perturbation Interrogation of the Internal and NearField Plasma Structure of a Hall Thruster Using a High-Speed Probe Positioning System," Ph.D. Dissertation, Dept. of Aerospace Engineering, Univ. of Michigan, Ann Arbor, MI, 2001.

${ }^{46}$ Choueiri, E. Y., "Plasma Oscillations in Hall Thrusters," Physics of Plasmas, Vol. 8, No. 4, 2001, pp. 1411-1426.

${ }^{47}$ Bishaev, A. M., and Kim, V., "Local Plasma Properties in a HallCurrent Accelerator with an Extended Acceleration Zone," Soviet PhysicsTechnical Physics, Vol. 23, No. 9, 1978, pp. 1055-1057.

${ }^{48}$ Azziz, Y., Warner, N. Z., Martinez-Sanchez, M., and Szabo, J. J., "High Voltage Plume Measurements and Internal Probing of the BHT-1000 Hall Thruster," AIAA Paper 2004-4097, July 2004.

\section{Bibliographies}

Garrigues, L., Hagelaar, G. J. M., Bareilles, J., Boniface, C., and Boeuf, J. P., "Model Study of the Influence of the Magnetic Field Configuration on the Performance and Lifetime of a Hall Thruster," Physics of Plasmas, Vol. 10, No. 12, 2003, pp. 4886-4892.

Jacobson, D. T., Manzella, D. H., Hofer, R. R., and Peterson, P. Y., "NASA's 2004 Hall Thruster Program," NASA TM-2004-213340, Oct. 2004.

Kim, V., Kozlov, V., Semenov, A., and Shkarban, I., "Investigation of the Boron Nitride Based Ceramics Sputtering Yield Under Its Bombardment by $\mathrm{Xe}$ and $\mathrm{Kr}$ Ions," 27th International Electric Propulsion Conf., Paper IEPC-01-073, Oct. 2001.

Manzella, D. H., Yim, J., and Boyd, I. D., "Predicting Hall Thruster Operational Lifetime," NASA TM-2004-213214, Aug. 2004.

Syage, J. A., "Electron-Impact Cross Sections for Multiple Ionization of Kr and Xe," Physical Review A: General Physics, Vol. 46, No. 9, 1992, pp. 5666-5679. 